\title{
Expression signatures of lncRNAs in skeletal muscles at the early flow phase revealed by microarray in burned rats
}

\author{
Zhang Haijun, M.D., Yu Yonghui, M.D., Chai Jiake, M.D. \\ Department of Burn and Plastic Surgery, First Affiliated Hospital of PLA General Hospital, Beijing, China
}

\begin{abstract}
BACKGROUND: Severe thermal trauma covering more than $30 \%$ of the total body surface area (TBSA) triggers a sustained pathophysiological response, which includes, but is not limited to, hypermetabolism, chronic inflammation, and severe skeletal muscle wasting. Long non-coding RNAs (IncRNAs) are an important class of pervasive genes involved in a variety of biological functions. However, the functions of IncRNAs in the regulation of responses of skeletal muscle wasting after severe burn have remained untested.
\end{abstract}

METHODS: Presently examined were the expression profiles of IncRNAs and messenger RNAs (mRNAs) in skeletal muscle tissues of 3 pairs of burned rats at the early flow phase, compared with sham rats, using microarray. Each potential IncRNA-mRNA pair identified is a strong candidate in the definitive confirmation of the presence of specific IncRNA-mRNA interactions, thus providing a detailed picture of the pathogenesis of skeletal muscle wasting in burned rats.

RESULTS: LncRNA expression levels were compared among 3 injured tissues and matched normal tissues from microarray data. An average of I I 7 significantly differentially expressed IncRNAs (I.5-fold) were identified. Only 202 mRNAs were significantly upregulated or downregulated, an average of 92 mRNAs were upregulated in injured, compared to matched normal, tissues, while an average of 110 mRNAs) were downregulated.

CONCLUSION: Presently identified were IncRNAs differentially expressed in skeletal muscles of burned rats, compared to normal tissues. Regulatory pathways may be involved in the pathogenesis of skeletal muscle wasting. Each IncRNA-mRNA pair identified is a strong candidate for a future study to definitively confirm the presence of specific IncRNA-mRNA interactions, thus providing a more detailed picture of the pathogenesis of skeletal muscle wasting in burned rats.

Keywords: Burn; expression; LncRNAs; microarray; muscle wasting.

\section{INTRODUCTION}

Injuries represent one of the most important public health problems in economically developing and developed countries. Of the major types of injuries, burns contribute to more than $1 \%$ of the global burden of disease. ${ }^{[1-3]}$ Burn injury is a primary cause of disability and mortality, with severe economic and social consequences. It can also lead to pain, as well as somatic and psychological complications. ${ }^{[1]}$ Severe thermal trauma covering more than $30 \%$ of total body surface area (TBSA) triggers a sustained pathophysiological response, which includes,

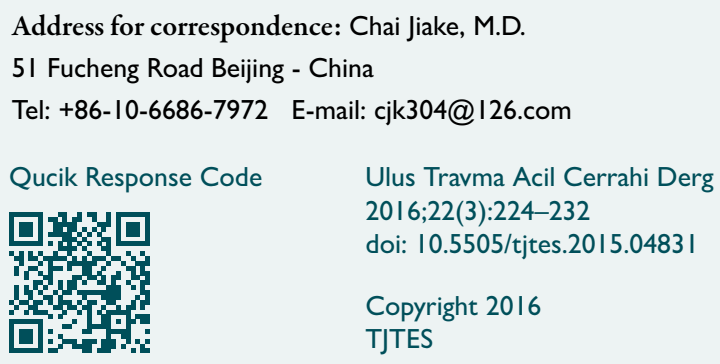

but is not limited to, hypermetabolism, chronic inflammation, marked elevations in peripheral catecholamines and cortisol levels, and severe skeletal muscle wasting. ${ }^{[2-4]}$

Severe burn injuries lead to a prolonged hypercatabolic state resulting in dramatic loss of skeletal muscle mass. ${ }^{[5]}$ As skeletal muscle accounts for over $50 \%$ of the dry weight of the body's cells, its catabolism exerts a profound effect on the body's metabolism as a whole. ${ }^{[6]}$ The primary mechanisms underlying skeletal muscle wasting induced by severe burn include activation of ubiquitin-proteasome pathway, ${ }^{[7,8]}$ myonuclear apoptosis, ${ }^{[9]}$ mitochondrial dysfunction, ${ }^{[10]}$ autophagy, ${ }^{[1]}$ signaling pathways driving muscle inflammation, and protein metabolism. ${ }^{[5]}$ Although the mechanisms of skeletal muscle wasting following severe burn are becoming increasingly clear, the mechanisms of transcriptional and/or post-transcriptional gene regulation remain unknown.

Long non-coding RNAs (IncRNAs) have gained widespread attention in recent years as a potentially new and crucial layer of biological regulation. ${ }^{[12]}$ LncRNAs have been implicated 
in a wide range of developmental processes and diseases. ${ }^{[13]}$ LncRNAs have emerged as key components of the address code, allowing protein complexes, genes, and chromosomes to be trafficked to appropriate locations, and subjected to proper activation and deactivation. LncRNA-based mechanisms control the fate of cells during development, and their dysregulation underlies some human disorders caused by chromosomal deletions and translocations. ${ }^{[14,15]}$

LncRNAs are generally longer than 200 nucleotides. Recent studies have shown that IncRNAs may act as important cis- or trans-regulators in various biological processes. ${ }^{[16]}$ Mutations in IncRNAs are associated with a wide range of diseases, including stress, and particularly cancers and neurodegenerative diseases. ${ }^{[17]}$ However, the profiles of burn-related IncRNAs have yet to be documented. Recent studies have shown that transcription of the mammalian genome is not only pervasive, but also enormously complex. It is estimated that an average of 10 transcription units, the vast majority of which make IncRNAs, may overlap with each traditional coding gene. These IncRNAs include antisense, and intronic and intergenic transcripts, as well as pseudogenes and retrotransposons. ${ }^{[8]}$

Presently examined are the expression profiles of IncRNAs and mRNAs in skeletal muscle tissues of 3 pairs of burned rats at the end of the shock phase, compared with sham rats, using microarray. Differentially expressed IncRNAs were then selected for target prediction with bioinformatics analyses. To improve the accuracy of target prediction, differentially expressed mRNAs were combined with target prediction of differentially expressed IncRNAs. The predicted target genes from the above analyses were subjected to bioinformatics analyses, ${ }^{[19]}$ including gene ontology analysis, pathway analysis, and network analysis. Analyzing potential molecular markers and the possible relationship between differentially expressed IncRNAs and protein-coding genes in skeletal muscles at the early flow phase in burned rats will provide further insights into the pathogenesis of skeletal muscle wasting in thermal trauma.

\section{MATERIALS AND METHODS}

\section{Sample Preparation}

The present study was approved by the Committee of Science and Technology of the First Affiliated Hospital of PLA General Hospital, in accordance with the protocol outlined in the Guide for the Care and Use of Laboratory Animals published by the US National Institutes of Health (NIH publication no. 85-23, revised 1996).

Six adult male Wistar rats weighing 200-220 g each were purchased from the Chinese Medical Scientific Institute (Beijing, China). Rats were kept in controlled standard housing conditions with free access to standard laboratory food and water for a 7-day adaptation period before being randomly assigned to groups.
After fur was clipped, a full thickness thermal injury of $30 \%$ of TBSA was inflicted by immersing the back of the trunk in $94^{\circ} \mathrm{C}$ water for 12 seconds. A weight- and time-matched sham-burn group $(n=3)$ was treated in the same manner as the trauma group $(n=3)$, with the exception of the immersion of the sham-burn animals in room temperature water. During the post-burn period and following immersion, all rats were immediately dried, administered fluid $(40 \mathrm{ml} / \mathrm{kg}$ of Ringer's lactate solution, calculated by the Parkland formula), and housed in individual cages with free access to food and water. ${ }^{[9]}$ On the third day following burn or sham injury, the animals were euthanized. Tibialis anterior muscles were harvested and stored at $-80^{\circ} \mathrm{C}$ for RNA extraction.

\section{LncRNA and mRNA Microarray}

Rat IncRNA array was designed for profiling IncRNA and protein-coding genes. Approximately 9,000 IncRNAs were selected from the NCBI Reference Sequence Database: all UCSC mRNA records and orthologs of rat IncRNAs. While probes for coding genes were printed once, probes for IncRNAs were printed 3 times. Probes for housekeeping genes and negative probes were printed multiple times to ensure hybridization quality.

\section{RNA Labeling and Array Hybridization}

Sample labeling and array hybridization were performed according to Agilent One-Color Microarray-Based Gene Expression Analysis Protocol (Agilent Technologies, Inc., Santa Clara, CA, USA). Briefly, total RNA from each sample was linearly amplified and labeled with Cy3-UTP. Labeled cRNAs were purified using the RNeasy Mini Kit (Qiagen, Inc., Hilden, Germany). Concentration and specific activity of labeled cRNAs (pmol Cy3/ $\mu$ g cRNA) were measured using the NanoDrop ND-1000 spectrophotometer (Thermo Fisher Scientific, Inc., Waltham, MA, USA). One $\mu \mathrm{g}$ of each labeled cRNA was fragmented by adding II $\mu$ of I0xblocking agent and 2.2 $\mu$ of $25 \times$ fragmentation buffer. The mixture was then heated at $60^{\circ} \mathrm{C}$ for $30 \mathrm{~min}$, and finally, $55 \mu \mathrm{l}$ of $2 \times \mathrm{GE}$ hybridization buffer was added to dilute the labeled cRNA. A total of 100 $\mu$ of hybridization solution was dispensed into the gasket slide and assembled onto the gene expression microarray slide. The slides were incubated at $65^{\circ} \mathrm{C}$ for 17 hours in an Agilent hybridization oven (Agilent Technologies, Inc., Santa Clara, CA, USA). The hybridized arrays were washed, fixed, and scanned using an Agilent DNA microarray scanner (part no. G2505C; Agilent Technologies, Inc., Santa Clara, CA, USA).

\section{Data Analysis}

Agilent Feature Extraction software (version I I.0.I.I; Agilent Technologies, Inc., Santa Clara, CA, USA) was used to analyze the acquired array images. Quantile normalization and subsequent data processing were performed using GeneSpring GX version 12.0 software package (Agilent Technologies, Inc., Santa Clara, CA, USA). Following quantile normalization of the raw data, IncRNAs and mRNAs for which at least 3 out 
of 6 samples had flags in "Present" or "Marginal" ("All Targets Value") were chosen for further data analysis.

Differentially expressed IncRNAs and mRNAs with statistical significance were identified using volcano plot filtering. Hierarchical clustering was performed using GeneSpring GX software (version 12.0; Agilent Technologies, Inc., Santa Clara, CA, USA).

\section{Construction of the Coding-Non-Coding Gene Co-Expression Network}

To show that the IncRNAs directly regulated the expression of target mRNAs, IncRNA target predictions were superimposed onto the IncRNA-mRNA correlation network. The resulting network was defined as an IncRNA-mRNA regulatory network. A direct connection was placed from an IncRNA to an mRNA using either a blue line (trans-interaction) or a red line (cis-interaction).

\section{Statistical Analysis}

Results were expressed as mean $\pm S D$. Statistical analysis was performed using Student's t-test for comparison of 2 groups in the microarray, and analysis of variance was used for multiple comparisons. In both cases, differences with $p<0.05$ were considered to be statistically significant. Statistical significance of microarray results was analyzed by fold change and Student's t-test. False discovery rate was calculated in order to correct $p$ values. The threshold value used to screen differentially expressed IncRNAs and mRNAs was a fold change $\geq 1.5(p<0.05)$

Table I. Upregulated IncRNA profiles

\begin{tabular}{|c|c|c|c|c|c|c|c|}
\hline \multirow[t]{2}{*}{ Probe name } & \multirow[t]{2}{*}{ p value } & \multicolumn{2}{|c|}{ Fold change and regulation } & \multirow[t]{2}{*}{ Probe name } & \multirow[t]{2}{*}{ p value } & \multicolumn{2}{|c|}{ Fold change and regulation } \\
\hline & & $\begin{array}{l}\text { Absolute fold change } \\
\text { ([T] vs }[\mathrm{C}])\end{array}$ & $\begin{array}{l}\text { Regulation } \\
\text { ([T] vs [C]) }\end{array}$ & & & $\begin{array}{l}\text { Absolute fold change } \\
\text { ([T] vs [C]) }\end{array}$ & $\begin{array}{l}\text { Regulation } \\
\text { ([T] vs }[C] \text { ) }\end{array}$ \\
\hline XR_007625_PI & 0.042436516 & 1.513741298 & up & S75669_PI & 0.007335414 & 1.939438731 & up \\
\hline XR_008295_PI & 0.006886517 & 1.608696682 & up & S75669_PI & $0.0073354 \mid 4$ & 1.939438731 & up \\
\hline MRAK0808I5_PI & 0.020947862 & 1.520309795 & up & S75669_PI & 0.007335414 & 1.939438731 & up \\
\hline XR_0055I5_PI & 0.040546402 & 1.626680595 & up & S75669_PI & 0.007335414 & I.93943873| & up \\
\hline XR_009083_PI & 0.006434708 & 1.617928104 & up & XR_009355_PI & 0.048364759 & $1.55660342 \mathrm{I}$ & up \\
\hline XR_006772_PI & 0.038080035 & 1.549397303 & up & XR_0055I3_PI & 0.006641351 & 1.75941563 & up \\
\hline XR_005733_PI & 0.01100545 & 1.551780677 & up & XR_00684I_PI & 0.028879691 & $1.56496150 \mathrm{I}$ & up \\
\hline XR_007062_PI & 0.003142118 & 1.914118172 & up & AY6435I4_PI & 0.033154207 & 1.503992702 & up \\
\hline XR_007206_PI & 0.024360851 & 2.084225919 & up & AY643514_PI & 0.033154207 & 1.503992702 & up \\
\hline MRAKI42335_PI & 0.043959593 & 1.955781634 & up & XR_006694_PI & 0.006682623 & 1.691703812 & up \\
\hline MRuc007guc_PI & 0.026266496 & 1.93846981 & up & MRAK05320I_PI & 0.049504017 & I.694329931 & up \\
\hline XR_006|48_PI & 0.012795552 & 1.640795758 & up & DQ832324_PI & 0.01193803 & 1.604519178 & up \\
\hline AF332363_PI & 0.009779 & I.76732704I & up & XR_008093_PI & 0.005252984 & 1.622082653 & up \\
\hline XR_007393_PI & 0.042234037 & 1.836920259 & up & XR_005488_PI & 0.049406948 & 1.739175513 & up \\
\hline MRAK040107_PI & 0.034288649 & 1.743452026 & up & XR_009I5I_PI & 0.034915223 & 1.514451804 & up \\
\hline MRAK040I07_PI & 0.034288649 & I.743452026 & up & XR_005800_PI & 0.001871706 & 1.715524821 & up \\
\hline BC098776_PI & 0.005731768 & 1.543092558 & up & uc.152-_PI & 0.04315685 & 1.713979119 & up \\
\hline XR_006678_PI & 0.042567859 & I.708809769 & up & XR_00850I_PI & 0.023420274 & 1.61734673 & up \\
\hline MRAKOI2222_PI & 0.018538406 & 1.592452676 & up & XR_007720_PI & 0.014669486 & 1.727730824 & up \\
\hline XR_006337_PI & 0.023330015 & 1.736155055 & up & XR_006550_PI & 0.002260132 & 1.531738302 & up \\
\hline XR_007784_PI & 0.010820094 & 1.541399506 & up & XR_005754_PI & 0.006875391 & 1.819631944 & up \\
\hline XR_006785_PI & 0.009177714 & I.647233223 & up & XR_00732I_PI & 0.011698519 & 1.673666406 & up \\
\hline MRAK006088_PI & 0.047430249 & 1.535065022 & up & XR_0089II_PI & 0.014100815 & 1.593491511 & up \\
\hline MRAK006088_PI & 0.047430249 & 1.535065022 & up & MRAK004I25_PI & 0.012228179 & 1.50735389 & up \\
\hline MRAK032396_PI & 0.002439049 & 1.745553893 & up & BCI68236_PI & 0.033712827 & 2.592948166 & up \\
\hline MRAK032396_PI & 0.002439049 & 1.745553893 & up & MRAK032II3_PI & 0.025968499 & 1.865618242 & up \\
\hline XR_006529_PI & 0.005485356 & 1.651250693 & up & XR_007247_PI & 0.013873027 & 1.574192895 & up \\
\hline MRAKI45223_PI & 0.028223934 & $|.90459084|$ & up & AY383698_PI & 0.042209102 & $|.52549| 35 \mid$ & up \\
\hline XR_006328_PI & 0.022030979 & 1.550920784 & up & AY383698_PI & 0.042209102 & 1.525491351 & up \\
\hline MRAKI35387_PI & 0.019185074 & 1.501364733 & up & MRAKI3I727_PI & 0.034235162 & 1.513193901 & up \\
\hline XR_005665_PI & 0.041147848 & 2.239151018 & up & XR_005772_PI & 0.035771322 & $1.533 \mid 47498$ & up \\
\hline S75669_PI & $0.0073354 \mid 4$ & $1.93943873 \mid$ & up & D26496_PI & 0.043220295 & 1.510179878 & up \\
\hline
\end{tabular}

\#Fold change cut-off: 1.5; \# $\mathrm{P}$ value cut-off: 0.05 ; \#Condition pairs: T vs C. 


\section{RESULTS}

\section{Overview of Expression Profiles of lncRNAs}

From the IncRNA expression profiles, differentially expressed IncRNAs were found from among samples of skeletal muscles obtained from thermal rats (thermal group) and sham-burn rats (control group). Expression profiles of IncRNAs in paired samples were shown by calculating logfold change of the thermal group/the control group (T/C). Agreement was formulated as follows: fold change cut-off was 1.5, and for any fold change, positive value indicated upregulation, while negative value indicated downregulation. Log-fold change signified $\log 2$ value of absolute fold change. Fold change and $p$ values were calculated from the normalized expression.

LncRNA expression levels were compared among the 3 injured tissues and their matched normal tissues from the microarray data, and an average of 117 long IncRNAs that were significantly differentially expressed ( 1.5 -fold) were identified.
Results demonstrated that a very large number of IncRNAs could be examined in normal and injured tissues, while only II7 of these were significantly upregulated (Table I) or downregulated (Table 2), and could be used to discriminate skeletal muscles of burned rats from matched normal tissues. Compared to the normal tissues, MRAK080917 (log2 fold change $\mathrm{T} / \mathrm{C}=6.777752082$ ) was most significantly downregulated, while $\mathrm{BCl} 68236$ (log2 fold change $\mathrm{T} / \mathrm{C}=2.592948 \mathrm{I} 66$ ) was most significantly upregulated. It was determined that downregulated IncRNAs were more common than those that were upregulated.

\section{Overview of mRNA Profiles}

Results demonstrated that tens of thousands of mRNAs could be examined in normal and injured tissues, but that only 202 mRNAs were significantly upregulated or downregulated. An average of 92 mRNAs were upregulated in the injured tissues (Table 3), compared to the matched normal ones, while an average of 110 mRNAs were downregulated (Table 4).

Table 2. Downregulated IncRNA profiles

\begin{tabular}{|c|c|c|c|c|c|c|c|}
\hline \multirow[t]{2}{*}{ Probe name } & \multirow[t]{2}{*}{ p value } & \multicolumn{2}{|c|}{ Fold change and regulation } & \multirow[t]{2}{*}{ Probe name } & \multirow[t]{2}{*}{ p value } & \multicolumn{2}{|c|}{ Fold change and regulation } \\
\hline & & $\begin{array}{l}\text { Absolute fold change } \\
\text { ([T] vs [C]) }\end{array}$ & $\begin{array}{l}\text { Regulation } \\
\text { ([T] vs [C]) }\end{array}$ & & & $\begin{array}{l}\text { Absolute fold change } \\
\text { ([T] vs [C]) }\end{array}$ & $\begin{array}{l}\text { Regulation } \\
\text { ([T] vs [C]) }\end{array}$ \\
\hline MRAKI5842 & $5.30947 \mathrm{E}-05$ & $2.39727 \mid 208$ & down & uc.414+_PI & 0.035614403 & 1.742281172 & down \\
\hline MRAK05384 & 0.047353911 & 1.826165856 & down & XR_008292_ & 0.002067679 & 1.683021803 & down \\
\hline BC088246_PI & 0.010256908 & 1.691622866 & down & BC088244_PI & 0.016885746 & 1.559107747 & down \\
\hline MRAK0I357 & 0.049684039 & 2.110840155 & down & XR_006390_ & 0.03336485 & 1.666808775 & down \\
\hline XR_005853_ & 0.012668374 & 1.995183722 & down & XR_007I $70_{-}$ & 0.031927225 & 1.504417159 & down \\
\hline XR_007420_ & 0.044076153 & 1.932416621 & down & MRAKOI40I & 0.047898874 & 1.861431079 & down \\
\hline MRAKI3343 & 0.046766231 & $1.74|47086|$ & down & BC09|43I_PI & 0.040444159 & 1.774696923 & down \\
\hline XR_008004_ & 0.049786366 & 1.564872337 & down & MRNR_0274 & 0.043534745 & 1.520794796 & down \\
\hline XR_007008_ & 0.027827377 & 1.586787642 & down & MRNR_0274 & 0.043534745 & 1.520794796 & down \\
\hline XR_009508_ & 0.010834758 & 1.621010085 & down & MRAK08854 & 0.034608972 & $1.59410648 \mid$ & down \\
\hline MRuc008lum & 0.046532415 & 1.731525939 & down & MRAKI3756 & 0.049387275 & 2.266269552 & down \\
\hline MRBC05287 & 0.036183082 & 1.986867961 & down & XR_006360_ & $0.03159504 \mid$ & 1.992246736 & down \\
\hline MRAKOI 247 & 0.035900857 & 1.627108736 & down & XR_00564I_ & 0.010076191 & 1.875123347 & down \\
\hline XR_006437_ & 0.035308453 & 1.655361222 & down & MRuc007lsc & 0.010926997 & 1.779102256 & down \\
\hline XR_006093_ & 0.044244767 & 1.578948628 & down & MRAK04093 & 0.033756148 & 2.464227295 & down \\
\hline L27I29_PI & 0.011395706 & 1.840498533 & down & MRAK08024 & 0.03462557 & 2.578548176 & down \\
\hline MRuc008ras & 0.043368383 & $|.65| 45436 \mid$ & down & uc.392+_PI & 0.030557302 & 1.897403964 & down \\
\hline XR_0084I5_ & 0.009885108 & 3.936073061 & down & XR_007260_ & 0.027345191 & 1.87398062 & down \\
\hline uc.292+_PI & 0.033380577 & 1.544869528 & down & BCI29II8_PI & 0.049050374 & 1.795345078 & down \\
\hline MRAK 16128 & 0.006865299 & 1.704064842 & down & BCI05769_PI & 0.036040215 & 1.532308552 & down \\
\hline BCI6188I_PI & 0.000525852 & 2.903724206 & down & MRBC0250I & $0.0288317 \mid$ & 1.84676539 & down \\
\hline MRAKI7259 & 0.047606367 & 1.505013888 & down & MRAK08082 & 0.003419968 & 1.688903112 & down \\
\hline XR_005949_ & 0.029279799 & 1.740566901 & down & BC091254_PI & 0.043449559 & 1.774148166 & down \\
\hline XR_006777_ & 0.044210245 & 1.586528935 & down & MRAK03323 & 0.022282206 & 2.550611731 & down \\
\hline MRAK0809I & 0.018362991 & 6.777752082 & down & MRAK08884 & 0.013406108 & 1.688892108 & down \\
\hline uc.414+_PI & 0.035614403 & 1.742281172 & down & MRAK037I8 & 0.018860453 & 2.452178953 & down \\
\hline uc.414+_PI & 0.035614403 & 1.742281172 & down & & & & \\
\hline
\end{tabular}

\#Fold change cut-off: 1.5; " $\mathrm{P}$ value cut-off: 0.05 ; \#Condition pairs: T vs C. 
Construction of the Coding-Non-Coding Gene

Co-Expression Network

A coding-non-coding gene co-expression network (CNC network) was constructed based on correlation analysis of the differentially expressed IncRNAs and mRNAs (Figure I). LncRNAs and mRNAs with Pearson's correlation coefficients not less than 0.99 were selected to draw the network, using the Cytoscape program (Institute of Systems Biology, Seattle,

Table 3. Upregulated mRNA profiles

\begin{tabular}{|c|c|c|c|c|c|c|c|}
\hline \multirow[t]{2}{*}{ Probe name } & \multirow[t]{2}{*}{ p value } & \multicolumn{2}{|c|}{ Fold change and regulation } & \multirow[t]{2}{*}{ Probe name } & \multirow[t]{2}{*}{$p$ value } & \multicolumn{2}{|c|}{ Fold change and regulation } \\
\hline & & $\begin{array}{l}\text { Absolute fold change } \\
\text { ([T] vs [C]) }\end{array}$ & $\begin{array}{l}\text { Regulation } \\
\text { ([T] vs }[\mathrm{C}] \text { ) }\end{array}$ & & & $\begin{array}{l}\text { Absolute fold change } \\
\text { ([T] vs [C]) }\end{array}$ & $\begin{array}{l}\text { Regulation } \\
\text { ([T] vs [C]) }\end{array}$ \\
\hline CUST_I210_PI421866198 & 0.045606394 & 1.59759874 & up & CUST_I1657_PI421866198 & 0.012815076 & 2.177136405 & up \\
\hline CUST_10195_PI421866198 & 0.010355438 & 5.063922528 & up & CUST_7560_PI421866198 & 0.040101847 & 1.65046247 & up \\
\hline CUST_I855_PI421866198 & 0.00137129 & 2.617241527 & up & CUST_10534_PI421866198 & 0.01945795 & 1.628197076 & up \\
\hline CUST_2486_PI421866198 & 0.020719392 & 1.90840495 & up & CUST_13435_PI421866198 & 0.037294419 & 2.018765962 & up \\
\hline CUST_4642_PI421866198 & 0.029708762 & 1.654661374 & up & CUST_3946_PI421866198 & 0.00503991 & 2.915539092 & up \\
\hline CUST_6944_PI421866198 & 0.007240272 & 4.257326589 & up & CUST_4654_PI421866198 & 0.001786365 & 3.507455905 & up \\
\hline CUST_15260_PI421866198 & 0.000577946 & 1.563042132 & up & CUST_10867_PI421866198 & 0.039276132 & 2.023074619 & up \\
\hline CUST_5342_PI421866198 & 0.037533375 & 2.430356579 & up & CUST_II542_PI421866198 & 0.001880145 & 3.595193945 & up \\
\hline CUST_II829_PI421866198 & 0.0100799 & 3.253144336 & up & CUST_874_PI421866198 & 0.030490411 & 1.549625107 & up \\
\hline CUST_15262_Pl421866198 & 0.00574348 & 2.040395513 & up & CUST_6327_PI421866198 & 0.019006624 & 1.656331373 & up \\
\hline CUST_2300_PI421866198 & 0.043473392 & 2.041859786 & up & CUST_10004_PI421866198 & 0.009444256 & 1.801275848 & up \\
\hline CUST_10432_PI421866198 & 0.014915171 & 3.255336544 & up & CUST_2643_PI421866198 & 0.042958202 & 1.611518406 & up \\
\hline CUST_7003_PI421866198 & 0.005680592 & 1.966802326 & up & CUST_I2088_PI421866198 & 0.007111663 & 4.217454975 & up \\
\hline CUST_5856_PI421866198 & 0.00444493 & 1.514130444 & up & CUST_II522_PI421866198 & 0.016947821 & $1.52648562 \mid$ & up \\
\hline CUST_3262_PI421866198 & 0.0111125493 & $1.62646867 \mid$ & up & CUST_7763_PI421866198 & 0.044559992 & 2.452112778 & up \\
\hline CUST_3486_PI421866198 & 0.026690711 & 1.861147332 & up & CUST_9248_PI421866198 & 0.021534995 & 1.573094493 & up \\
\hline CUST_4557_PI421866198 & 0.032782771 & $2.3774 \mid 8854$ & up & CUST_I099I_PI421866198 & 0.033401846 & 2.651287522 & up \\
\hline CUST_13837_PI421866198 & 0.036516598 & 1.518998312 & up & CUST_654_PI421866198 & 0.011979725 & 1.904043361 & up \\
\hline CUST_7855_PI421866198 & 0.013731288 & 2.031357834 & up & CUST_II606_PI421866198 & 0.035018534 & 2.611823367 & up \\
\hline CUST_9330_PI421866198 & 0.002192444 & 1.821304992 & up & CUST_I4500_PI421866198 & 0.011436587 & 2.178194373 & up \\
\hline CUST_8448_PI421866198 & 0.03433747 & 2.348021686 & up & CUST_1791_PI421866198 & 0.012327838 & 1.685268466 & up \\
\hline CUST_7759_PI421866198 & 0.030562675 & 2.302327658 & up & CUST_10895_PI421866198 & 0.002460083 & 1.803186767 & up \\
\hline CUST_8107_PI421866198 & 0.039949858 & 1.817725142 & up & CUST_2059_PI421866198 & 0.016699039 & 4.0862396 & up \\
\hline CUST_13967_PI421866198 & 0.011714172 & 1.502536808 & up & CUST_I2802_PI421866198 & 0.001146504 & 1.959951788 & up \\
\hline CUST_I3858_PI42I866I98 & 0.031802356 & $4.327 \mid 22709$ & up & CUST_14787_PI421866198 & 0.021016027 & 1.847760579 & up \\
\hline CUST_3094_PI421866198 & 0.049342616 & 1.559352399 & up & CUST_I2797_PI421866198 & 0.043673356 & 1.626239603 & up \\
\hline CUST_483I_PI421866198 & 0.009792921 & 3.028942659 & up & CUST_3027_PI421866198 & 0.038742155 & 2.095886609 & up \\
\hline CUST_9367_PI421866198 & 0.002270001 & 1.504368323 & up & CUST_3860_PI421866198 & 0.006603383 & 2.072462648 & up \\
\hline CUST_I2576_PI421866198 & 0.021936529 & 1.530467349 & up & CUST_9836_PI421866198 & 0.046263826 & 2.805332937 & up \\
\hline CUST_II559_PI421866198 & 0.037735196 & 1.586725757 & up & CUST_6100_PI421866198 & 0.023983273 & 1.646977676 & up \\
\hline CUST_10215_PI421866198 & 0.020469822 & 1.987755392 & up & CUST_9174_PI421866198 & 0.044585762 & 1.572939157 & up \\
\hline CUST_862_PI421866198 & 0.029139124 & 1.62620372 & up & CUST_9559_PI421866198 & 0.00320455 & 1.85037819 & up \\
\hline CUST_II044_PI421866198 & 0.043897171 & 2.412127444 & up & CUST_I2615_PI421866198 & 0.044961697 & 5.12824374 & up \\
\hline CUST_II8I5_PI421866198 & 0.008441526 & 3.050925744 & up & CUST_12694_PI421866198 & 0.000445438 & 6.028735911 & up \\
\hline CUST_12246_PI421866198 & 0.03359414 & 1.950991285 & up & CUST_5146_PI421866198 & 0.029780754 & 3.435343433 & up \\
\hline CUST_I3854_PI421866198 & 0.042522872 & $2.03757764 \mid$ & up & CUST_14214_PI421866198 & 0.022183257 & 1.906633522 & up \\
\hline CUST_8809_PI421866198 & 0.017832572 & 2.054859613 & up & CUST_5814_PI421866198 & 0.019676952 & 2.119471646 & up \\
\hline CUST_27I0_PI421866198 & 0.002845954 & 8.341076172 & up & CUST_5887_PI421866198 & 0.04427284 & 1.726615686 & up \\
\hline CUST_3992_PI421866198 & $0.03737254 \mid$ & $|.58423944|$ & up & CUST_9879_PI421866198 & 0.026613386 & 3.480238877 & up \\
\hline CUST_3166_PI421866198 & 0.00670917 & 1.673006325 & up & CUST_10303_PI421866198 & 0.030728741 & 4.236377053 & up \\
\hline CUST_6053_PI421866198 & $0.04609347 \mathrm{I}$ & $1.7312587 \mid 5$ & up & CUST_5232_PI421866198 & 0.019294662 & 1.711679983 & up \\
\hline CUST_II23I_PI42I866I98 & 0.036003194 & $|.66504442|$ & up & CUST_539_PI421866198 & 0.034040423 & 2.229217679 & up \\
\hline CUST_7773_PI421866198 & 0.036907358 & 1.608969152 & up & CUST_1893_PI421866198 & 0.010545646 & 1.566298301 & up \\
\hline CUST_8733_PI421866198 & 0.015020579 & 1.695315831 & up & CUST_2513_PI421866198 & 0.02086206 & 2.028827679 & up \\
\hline CUST_6940_PI421866198 & 0.036482366 & 1.644952586 & up & CUST_2838_PI421866198 & 0.019319005 & 1.660433334 & up \\
\hline CUST_|492_PI421866198 & 0.037677048 & 1.765124567 & up & CUST_370_PI421866198 & 0.000366622 & $1.907 \mid 48258$ & up \\
\hline
\end{tabular}


Table 4. Downregulated mRNA profiles

\begin{tabular}{|c|c|c|c|c|c|c|c|}
\hline \multirow[t]{2}{*}{ Probe name } & \multirow[t]{2}{*}{ p value } & \multicolumn{2}{|c|}{ Fold change and regulation } & \multirow[t]{2}{*}{ Probe name } & \multirow[t]{2}{*}{ p value } & \multicolumn{2}{|c|}{ Fold change and regulation } \\
\hline & & $\begin{array}{l}\text { Absolute fold change } \\
\text { ([T] vs }[\mathrm{C}])\end{array}$ & $\begin{array}{l}\text { Regulation } \\
\text { ([T] vs [C]) }\end{array}$ & & & $\begin{array}{l}\text { Absolute fold change } \\
\text { ([T] vs [C]) }\end{array}$ & $\begin{array}{l}\text { Regulation } \\
\text { ([T] vs [C]) }\end{array}$ \\
\hline CUST_4198_PI421866198 & 0.0067783 & 1.628791683 & down & CUST_7536_PI421866198 & 0.049372269 & 1.895256483 & down \\
\hline CUST_776_PI421866198 & 0.007212239 & 1.814502444 & down & CUST_9125_PI421866198 & 0.012439588 & 2.087408269 & down \\
\hline CUST_10318_PI421866198 & 0.02131578 & 3.252387526 & down & CUST_I1390_PI421866198 & 0.048161951 & 2.326862939 & down \\
\hline CUST_7779_PI421866198 & 0.033695322 & 1.75749043 & down & CUST_10258_PI421866198 & 0.037506169 & 1.684580066 & down \\
\hline CUST_3858_PI421866198 & 0.003915407 & 1.539287448 & down & CUST_II90I_PI421866198 & $0.03|87064|$ & 1.941909095 & down \\
\hline CUST_1082_PI421866198 & 0.029269283 & 1.585292074 & down & CUST_3360_PI421866198 & 0.024288361 & 1.964775215 & down \\
\hline CUST_4192_PI421866198 & 0.013624005 & 1.502800985 & down & CUST_3248_PI421866198 & 0.01717798 & 1.65095485 & down \\
\hline CUST_14720_PI421866198 & 0.044151095 & 1.983600187 & down & CUST_II572_PI421866I98 & 0.042170403 & 1.623829696 & down \\
\hline CUST_10036_PI421866198 & 0.015626469 & 1.605582355 & down & CUST_I3054_PI421866198 & 0.010350555 & 2.672858999 & down \\
\hline CUST_5668_PI421866198 & 0.008412707 & $1.54909 \mid 469$ & down & CUST_9107_PI421866198 & 0.039358033 & 2.054262673 & down \\
\hline CUST_II039_PI421866198 & $0.02234 I 275$ & 4.302450915 & down & CUST_10023_PI421866198 & 0.033561008 & 1.872143645 & down \\
\hline CUST_I2190_PI421866198 & 0.038150892 & 2.184822485 & down & CUST_857I_PI421866198 & 0.024507169 & 1.931788968 & down \\
\hline CUST_7670_PI421866198 & 0.030325542 & 1.786994631 & down & CUST_6493_PI421866198 & 0.016041024 & 1.534768436 & down \\
\hline CUST_64II_PI421866I98 & 0.02170252 & 4.228192419 & down & CUST_4967_PI421866198 & 0.016405071 & 1.732526997 & down \\
\hline CUST_9295_PI421866198 & 0.012044678 & 5.265246349 & down & CUST_9652_PI421866198 & 0.038653891 & 1.698414312 & down \\
\hline CUST_333_PI421866198 & 0.02729877 & 2.068126692 & down & CUST_II024_PI421866198 & 0.041375285 & 1.585211494 & down \\
\hline CUST_723I_PI421866198 & 0.045267906 & 1.988425486 & down & CUST_I1380_PI421866198 & 0.023145073 & 1.972866179 & down \\
\hline CUST_2560_PI421866198 & 0.049736128 & 2.029461915 & down & CUST_4567_PI421866198 & 0.000735864 & 1.609649972 & down \\
\hline CUST_33_PI421866198 & 0.014357628 & 1.634448213 & down & CUST_8200_PI421866198 & 0.015255319 & 1.854687725 & down \\
\hline CUST_9028_PI421866198 & 0.003902698 & 1.500420452 & down & CUST_5928_Pl421866198 & 0.034725016 & 1.649610019 & down \\
\hline CUST_|4308_PI421866198 & 0.007826484 & 1.599796943 & down & CUST_|4935_PI421866198 & 0.012652676 & 1.777394587 & down \\
\hline CUST_8516_PI421866198 & 0.026925455 & I.545754637 & down & CUST_8147_PI421866198 & 0.047774903 & 2.512465466 & down \\
\hline CUST_3482_PI421866198 & 0.027384736 & 1.56126868 & down & CUST_II318_PI421866198 & 0.024469389 & 1.801610366 & down \\
\hline CUST_6919_PI421866198 & 0.023519185 & 2.867930507 & down & CUST_12570_PI421866198 & 0.0199879 & 6.164450222 & down \\
\hline CUST_2509_PI421866198 & 0.041171198 & 2.197500256 & down & CUST_9797_PI421866198 & 0.018976082 & 2.905925735 & down \\
\hline CUST_5357_PI421866198 & 0.005533369 & 2.464244091 & down & CUST_I3012_PI421866198 & 0.044403789 & 1.566228458 & down \\
\hline CUST_383_PI421866198 & $0.0058994 \mid 4$ & 2.043783909 & down & CUST_2160_PI421866198 & 0.023758555 & 1.875550749 & down \\
\hline CUST_6065_PI421866198 & 0.033150212 & 3.01190688 & down & CUST_6349_PI421866198 & 0.009680624 & 1.664260649 & down \\
\hline CUST_II240_PI421866I98 & 0.009535117 & 1.604777926 & down & CUST_609_PI421866198 & 0.039611572 & 2.048383439 & down \\
\hline CUST_5578_PI421866198 & 0.022595551 & 1.518187137 & down & CUST_509I_PI421866198 & 0.029473561 & 1.741826508 & down \\
\hline CUST_I0I_PI421866198 & 0.024100642 & 1.732010889 & down & CUST_9434_PI421866198 & 0.016207278 & 1.699652485 & down \\
\hline CUST_I302_PI421866198 & 0.010298785 & 3.640830473 & down & CUST_14233_PI421866198 & 0.025889633 & 1.830465522 & down \\
\hline CUST_839I_PI421866198 & 0.019406484 & 1.678849454 & down & CUST_1862_PI421866198 & 0.043523032 & 1.989012487 & down \\
\hline CUST_4319_PI421866198 & 0.018043175 & 1.591434007 & down & CUST_2828_PI421866198 & 0.012457514 & 2.296501406 & down \\
\hline CUST_55I4_PI421866198 & 0.021748994 & 2.160062964 & down & CUST_3303_PI421866198 & $0.00078504 \mid$ & 1.634238713 & down \\
\hline CUST_I2018_PI421866198 & 0.043888152 & 2.505456518 & down & CUST_7885_PI421866198 & 0.019408921 & 1.61555388 & down \\
\hline CUST_|4802_PI421866198 & 0.047467746 & 2.091477871 & down & CUST_6904_PI421866198 & 0.031971115 & 1.687945512 & down \\
\hline CUST_1284I_PI42I866198 & 0.015876313 & 2.924250563 & down & CUST_9084_PI421866198 & 0.020976626 & 1.633773816 & down \\
\hline CUST_4276_PI421866198 & 0.037379472 & $1.54 \mid 802423$ & down & CUST_10372_PI421866198 & 0.034163437 & 1.531920752 & down \\
\hline CUST_4886_PI421866198 & 0.028589317 & 1.645704145 & down & CUST_6343_PI421866198 & $0.0235077 \mid$ & 1.591532443 & down \\
\hline CUST_7724_PI421866198 & 0.026010169 & 1.700709814 & down & CUST_9900_Pl421866198 & 0.009545992 & $1.627704 \mid 49$ & down \\
\hline CUST_1736_PI421866198 & 0.034807531 & 1.527727959 & down & CUST_2923_PI421866198 & 0.01879922 & 1.526455819 & down \\
\hline CUST_3184_PI421866198 & 0.027050956 & 1.67183407 & down & CUST_34|4_PI421866198 & 0.045886349 & I.728345527 & down \\
\hline CUST_560I_PI421866198 & 0.000686672 & 1.52572276 & down & CUST_9269_PI421866198 & 0.030872404 & I.79546|85। & down \\
\hline CUST_584_PI421866198 & 0.044594505 & 1.623093751 & down & CUST_1731_PI421866198 & 0.004534508 & 1.519119469 & down \\
\hline CUST_12238_PI421866198 & 0.001505492 & 1.510598473 & down & CUST_14236_PI421866198 & 0.000522689 & 2.343916593 & down \\
\hline CUST_6202_PI421866198 & 0.034681376 & 1.680710099 & down & CUST_|4943_PI421866198 & 0.041784213 & 1.52157488 & down \\
\hline CUST_5030_PI421866198 & 0.047914238 & 1.735625876 & down & CUST_9835_Pl421866198 & 0.009566858 & 1.549027046 & down \\
\hline CUST_|4123_PI421866198 & 0.00919136 & 1.793223113 & down & CUST_1976_PI421866198 & 0.037596345 & 1.768400442 & down \\
\hline CUST_5098_PI421866198 & 0.037846355 & 1.676871727 & down & CUST_5440_PI421866198 & 0.003370002 & 1.966515649 & down \\
\hline CUST_12249_PI421866198 & 0.035589358 & 1.693185845 & down & CUST_60I_PI42I866198 & 0.008108411 & $\mathrm{I} .546657228$ & down \\
\hline CUST_|4318_PI421866198 & 0.039627075 & 1.887104087 & down & CUST_596_PI421866198 & 0.03101802 & 1.518329418 & down \\
\hline CUST_12907_PI42I866198 & 0.030763082 & 1.553773667 & down & CUST_8394_PI421866198 & 0.020034374 & 1.596096633 & down \\
\hline CUST_6039_PI421866198 & 0.047285771 & 1.537592638 & down & CUST_8563_PI421866198 & 0.027774993 & 2.714663103 & down \\
\hline CUST_19_PI421866198 & 0.042225177 & $1.83289|77|$ & down & CUST_6633_PI421866198 & 0.006083932 & 1.746328538 & down \\
\hline
\end{tabular}




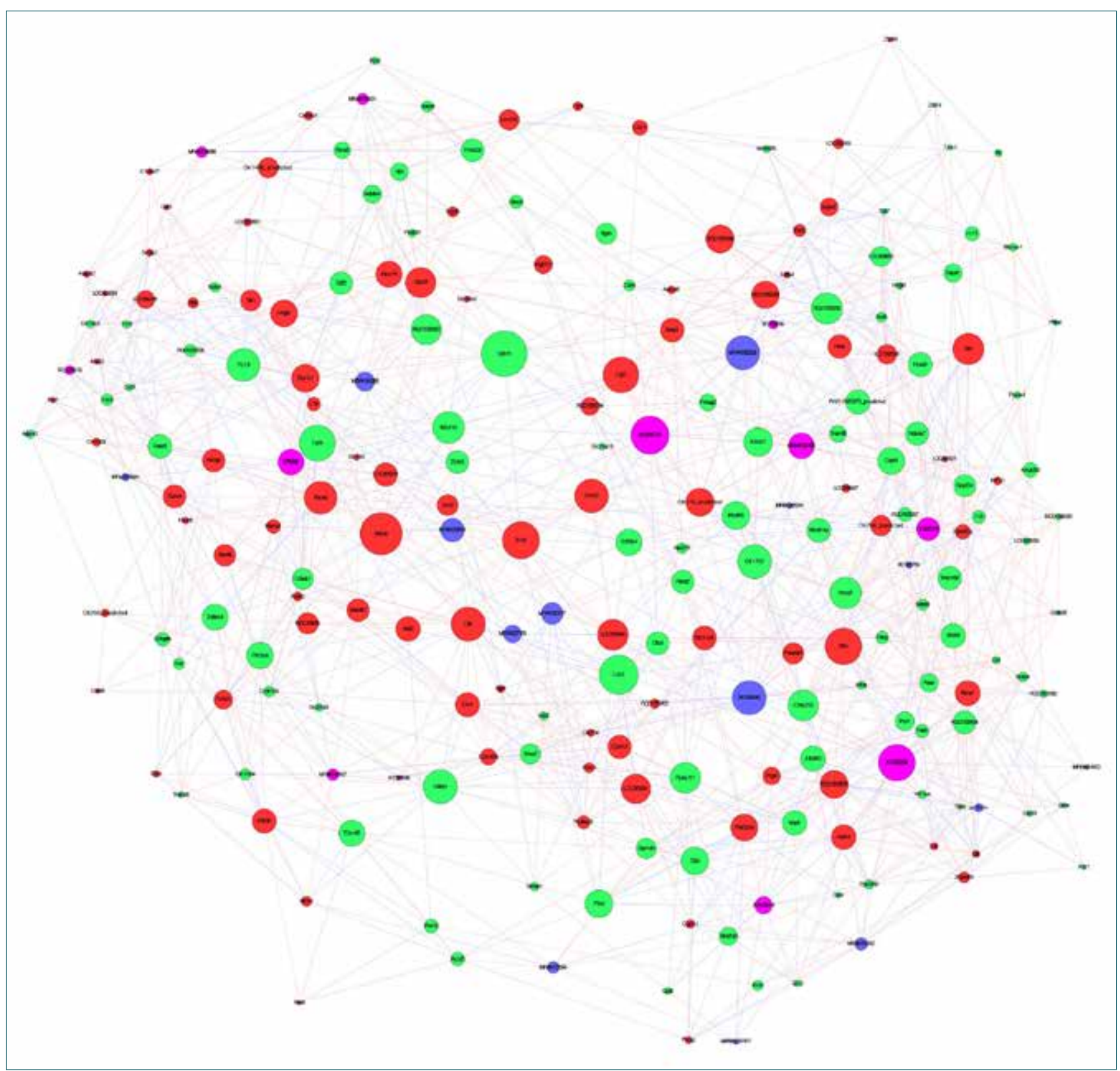

Figure 1. Predicted regulation network. A coding-non-coding gene co-expression network.

Node color: Upregulated IncRNAs: purple; Downregulated IncRNAs: blue; Upregulated mRNAs: red; Downregulated mRNAs: green.

Node size: Betweenness centrality (equal to the number of shortest paths from all vertices to all others that pass through that node).

Line color: Trans interaction: blue; Cis interaction: red; Cut-off: abs(correlation) $>=0.95$.

WA, USA); 117 IncRNAs and 202 mRNAs comprised the CNC network node. A total of 319 network nodes made 1392 associated network pairs of co-expressed IncRNAs and mRNAs. The CNC network indicated that one mRNA could correlate with I to several IncRNAs, and vice versa. The CNC network presented in Table S9 could implicate the inter-regulation of IncRNAs and mRNAs in burned rats.

\section{DISCUSSION}

Skeletal muscle wasting is an exacerbating factor in the prognosis of critically ill patients, including those with severe burn injury. ${ }^{\left[{ }^{[1]}\right]}$ Molecular treatment mechanisms of skeletal muscle wasting in burn injuries have been extensively studied. However, the pathogenesis and gene regulation involved are still unknown.

Increasing evidence has confirmed IncRNAs to be one of the most important factors controlling gene expression. [20] Therefore, the IncRNA expression profile in the skeletal muscle tissue of burned rats was presently evaluated in an attempt to reveal the potential role of IncRNAs in the pathogenesis of skeletal muscle wasting in burn injury (Tables I, 2). Microarray analyses revealed a set of differentially expressed IncRNAs, with 64 upregulated and 53 downregulated 
IncRNAs in skeletal muscles of burned rats, when compared to normal tissue.

Recent studies have demonstrated that IncRNAs can guide changes in gene expression in either the cis (neighboring genes) or trans (distantly located genes) manner, which is not easily predicted, based on IncRNA sequence. ${ }^{[1]}$ In principle, IncRNAs can guide chromatin change in cis in a cotranscriptional manner (tethered by RNA polymerase) or as a complementary target for small regulatory RNAs. Guidance in trans can occur by an IncRNA binding to target DNA as an RNA:DNA heteroduplex, RNA:DNA:DNA triplex, or by RNA recognition of a complex surface of specific chromatin features. ${ }^{[22]}$

The established role of IncRNAs in diseases creates an urgency to understand the mechanisms by which these RNAs seek their targets. A simplistic model in which the RNA remains tethered to the site of origin to regulate transcriptional changes in cis has been suggested. Perhaps the most intensely studied and best understood cis mechanism of regulation by IncRNA is the mammalian $X$ inactivation center, a genetic locus that specifies a number of IncRNAs, including $X$-inactivation-specific transcript, or XIST. ${ }^{[2]}$ Target genes under cis mechanism of regulation by IncRNAs were predicted via genome browser and annotation tool.

Further investigation of the IncRNA-gene network in an effort to gain insight into the functions of IncRNA targets demonstrated that MRAK080917 was predicted to target the Zbtbl 6 genes of the glioma pathway, which could affect total body weight, adiposity, lipid profile, insulin sensitivity of skeletal muscles, and mitochondrial function in skeletal muscles. ${ }^{[23,24]}$ Uc.4I4+ was predicted to target THRA and NrIdI. THRA is downregulated in skeletal muscle of patients with non-thyroidal illness syndrome secondary to non-septic shock, ${ }^{[25]}$ while $\mathrm{NrIdI}$ could modulate skeletal muscle oxidative capacity by regulating mitochondrial biogenesis and autophagy. ${ }^{[26]}$ MRBC025014 was predicted to target Sucla2, which is involved in mitochondrial disorder and progressive dystonia. ${ }^{[27,28]}$

\section{Conclusions}

In conclusion, IncRNAs differentially expressed in skeletal muscles of burned rats, compared to normal tissues, were identified in the present study. Regulatory pathways of IncRNAs may be involved in the pathogenesis of skeletal muscle wasting. Moreover, MRAK0809I7, uc.4I4+, and MRBC0250I4 could be critical in skeletal muscle wasting, via their targets, but these findings need to be confirmed, and the underlying mechanisms require further study. The present results also point to several exciting directions for future research. The CNC network presented in Table S9 implicated the inter-regulation of IncRNAs and mRNAs in burned rats. Each potential IncRNA-mRNA pair identified is a strong candidate for a future study that can definitively confirm the presence of specific IncRNA-mRNA interactions, ${ }^{[29]}$ thus providing a more detailed picture of the pathogenesis of skeletal muscle wasting in burned rats.

\section{Acknowledgments}

This study was financially supported by the National Natural Science Foundation of China (8II2010804I, 8147I873, $81171807)$ and the State Key Program of the General Logistics Department of the PLA (BWSI4J048, BWSI4J049). The authors wish to thank Kang Cheng Bio-tech Shanghai P.R. China for the microarray work.

Conflict of interest: None declared.

\section{REFERENCES}

1. Mohammadi-Barzelighi H1, Alaghehbandan R, Motevallian A, Alinejad F, Soleimanzadeh-Moghadam S, Sattari M, et al. Epidemiology of severe burn injuries in a Tertiary Burn Centre in Tehran, Iran. Ann Burns Fire Disasters 2011;24:59-62.

2. Porter C, Hurren NM, Herndon DN, Børsheim E. Whole body and skeletal muscle protein turnover in recovery from burns. Int J Burns Trauma 2013;3:9-17.

3. Jeschke MG, Gauglitz GG, Kulp GA, Finnerty CC, Williams FN, Kraft $\mathrm{R}$, et al. Long-term persistance of the pathophysiologic response to severe burn injury. PLoS One. 2011;6:e21245. Crossref

4. Chai J, Wu Y, Sheng ZZ. Role of ubiquitin-proteasome pathway in skeletal muscle wasting in rats with endotoxemia. Crit Care Med 2003;31:1802-7. Crossref

5. Merritt EK, Cross JM, Bamman MM. Inflammatory and protein metabolism signaling responses in human skeletal muscle after burn injury. $\mathrm{J}$ Burn Care Res 2012;33:291-7. Crossref

6. Cha1 JK. Mechanisms of skeletal muscle wasting after severe burn and its treatment. [Article in Chinese] Zhonghua Shao Shang Za Zhi 2009;25:243-5.

7. Lagirand-Cantaloube J, Cornille K, Csibi A, Batonnet-Pichon S, Leibovitch MP, Leibovitch SA. Inhibition of atrogin-1/MAFbx mediated MyoD proteolysis prevents skeletal muscle atrophy in vivo. PLoS One 2009;4:e4973. Crossref

8. Sheriff S, Kadeer N, Joshi R, Friend LA, James JH, Balasubramaniam A. Des-acyl ghrelin exhibits pro-anabolic and anti-catabolic effects on C2C12 myotubes exposed to cytokines and reduces burn-induced muscle proteolysis in rats. Mol Cell Endocrinol 2012;351:286-95. Crossref

9. Duan H, Chai J, Sheng Z, Yao Y, Yin H, Liang L, et al. Effect of burn injury on apoptosis and expression of apoptosis-related genes/proteins in skeletal muscles of rats. Apoptosis 2009;14:52-65. Crossref

10. Tzika AA, Mintzopoulos D, Mindrinos M, Zhang J, Rahme LG, Tompkins RG. Microarray analysis suggests that burn injury results in mitochondrial dysfunction in human skeletal muscle. Int J Mol Med 2009;24:387-92. Crossref

11. Hosokawa S, Koseki H, Nagashima M, Maeyama Y, Yomogida K, Mehr $\mathrm{C}$, et al. Title efficacy of phosphodiesterase 5 inhibitor on distant burninduced muscle autophagy, microcirculation, and survival rate. Am J Physiol Endocrinol Metab 2013;304:922-33. Crossref

12. Zhang J, Cui X, Shen Y, Pang L, Zhang A, Fu Z, et al. Distinct expression profiles of LncRNAs between brown adipose tissue and skeletal muscle. Biochem Biophys Res Commun 2014;443:1028-34. Crossref

13. Batista PJ, Chang HY. Long noncoding RNAs: cellular address codes in development and disease. Cell 2013;152:1298-307. Crossref 
14. Kung JT, Colognori D, Lee JT. Long noncoding RNAs: past, present, and future. Genetics 2013;193:651-69. Crossref

15. Lee JT, Bartolomei MS. X-inactivation, imprinting, and long noncoding RNAs in health and disease. Cell 2013;152:1308-23. Crossref

16. Ma H, Hao Y, Dong X, Gong Q, Chen J, Zhang J, et al. Molecular mechanisms and function prediction of long noncoding RNA. ScientificWorldJournal 2012;2012:541786. Crossref

17. Mizutani R, Wakamatsu A, Tanaka N, Yoshida H, Tochigi N, Suzuki $\mathrm{Y}$, et al. Identification and characterization of novel genotoxic stress-inducible nuclear long noncoding RNAs in mammalian cells. PLoS One 2012;7:e34949. Crossref

18. Lee JT. Epigenetic regulation by long noncoding RNAs. Science 2012;338:1435-9. Crossref

19. Li JP, Liu LH, Li J, Chen Y, Jiang XW, Ouyang YR, et al. Microarray expression profile of long noncoding RNAs in human osteosarcoma. Biochem Biophys Res Commun 2013;433:200,6.

20. Khachane AN, Harrison PM. Mining mammalian transcript data for functional long non-coding RNAs. PLoS One 2010;5:e10316. Crossref

21. Hung T, Chang HY. Long noncoding RNA in genome regulation: prospects and mechanisms. RNA Biol 2010;7:582-5. Crossref

22. Pauli A, Rinn JL, Schier AF. Non-coding RNAs as regulators of embryogenesis. Nat Rev Genet 2011;12:136-49. Crossref

23. Seda O, Liska F, Sedová L, Kazdová L, Krenová D, Kren V. A 14-gene region of rat chromosome 8 in SHR-derived polydactylous congenic substrain affects muscle-specific insulin resistance, dyslipidaemia and visceral adiposity. Folia Biol (Praha) 2005;51:53-61.

24. Plaisier CL, Bennett BJ, He A, Guan B, Lusis AJ, Reue K, et al. Zbtb16 has a role in brown adipocyte bioenergetics. Nutr Diabetes 2012;2:e46.

25. Lado-Abeal J, Romero A, Castro-Piedras I, Rodriguez-Perez A, AlvarezEscudero J. Thyroid hormone receptors are down-regulated in skeletal muscle of patients with non-thyroidal illness syndrome secondary to nonseptic shock. Eur J Endocrinol 2010;163:765-73. Crossref

26. Woldt E, Sebti Y, Solt LA, Duhem C, Lancel S, Eeckhoute J, et al. Reverb- $\alpha$ modulates skeletal muscle oxidative capacity by regulating mitochondrial biogenesis and autophagy. Nat Med 2013;19:1039-46. Crossref

27. Randolph LM, Jackson HA, Wang J, Shimada H, Sanchez-Lara PA, Wong DA, et al. Fatal infantile lactic acidosis and a novel homozygous mutation in the SUCLG1 gene: a mitochondrial DNA depletion disorder. Mol Genet Metab 2011;102:149-52. Crossref

28. Morava E, Steuerwald U, Carrozzo R, Kluijtmans LA, Joensen F, Santer $\mathrm{R}$, et al. Dystonia and deafness due to SUCLA2 defect; Clinical course and biochemical markers in 16 children. Mitochondrion 2009;9:43842. Crossref

29. Yu H, Kim PM, Sprecher E, Trifonov V, Gerstein M. The importance of bottlenecks in protein networks: correlation with gene essentiality and expression dynamics. PLoS Comput Biol 2007;3:e59. Crossref

\section{DENEYSEL ÇALIŞMA - ÖZET}

\section{Yanık sıçanlarda mikrodizi analiziyle ortaya çıkartıldığı gibi erken akış fazında iskelet kaslarındaki IncRNA'ların ekspresyon imzaları}

\section{Dr. Zhang Haijun, Dr. Yu Yonghui, Dr. Chai Jiake}

PLA Genel Hastanesi, Yanık ve Plastik Cerrahi Kliniği, Pekin, Çin

AMAÇ: Tüm vücut yüzeyinin (TVY) \%30'dan fazlasını kaplayan ağır termal travma hipermetabolizma, kronik enflamasyon ve iskelet kaslarında güçsüzleşmeyi içermekle birlikte bu bulgularla sınırlı olmayan süregelen bir fizyopatolojik yanıtı da tetiklemektedir. Uzun zincirli proteini kodlamayan RNA'lar (IncRNA'lar) çeşitli biyolojik fonksiyonlara katılan, önemli ve yaygın bgenler sınıfıdır. Ancak ağır yanıklardan sonra iskelet kaslarının zayıflamasına ilişkin yanıtları düzenleyen RNA'ların fonksiyonları hâlâ test edilmemiştir.

GEREÇ VE YÖNTEM: Burada mikrodizi analizi kullanılarak, plasebo sıçanlarla karşılaştırmalı olarak erken akış fazında üç çift yanık sıçanın iskelet kası dokularındaki IncRNA'lar ve haberci RNA'ların (miRNA'lar) ekspresyon profilleri incelendi. Tanımlanmış her bir potansiyel IncRNA-mRNA çifti spesifik IncRNA-mRNA etkileşimlerin varlığının kesin olarak doğrulanmasında kullanılan ve yanık sıçanlarda iskelet kası zayıflamasının ayrıntılı bir patogenezini gösteren güçlü bir aday belirteçtir.

BULGULAR: Üç yaralı dokuyla eşleştirilmiş üç normal doku örneğinde mikrodizi analiz verileri kullanılarak LncRNA ekspresyon düzeyleri karşılaştıııldı. Ortalama 1 I7 adet anlamlı derecede farklılaşmış IncRNA ( I.5 katı) tanımlandı. Yalnızca 202 adet miRNA anlamlı derecede arttı veya azaldı. Eşleştirilmiş normal dokulara göre zedelenmiş dokularda ortalama 92 adet miRNA'nın düzeyleri artarken, ortalama II0 miRNA'nın düzeyleri azaldı.

TARTIŞMA: Burada, normal dokulara göre sıçanların yanık dokularında IncRNA'ların farklı düzeylerde eksprese edildiği saptanmış̧ır. İskelet kasları zayıflamasının patogenezinde düzenleyici yolaklar da rol oynayabilmektedir. Tanımlanmş her bir IncRNA-mRNA çifti spesifik IncRNA-miRNAA etkileşimlerinin varlığııın doğrulanmasında kullanılan ve yanık sıçanlarda iskelet kası zayıflamasının ayrıntılı bir patogenezini gösteren güçlü bir aday belirteçtir.

Anahtar sözcükler: Ekspresyon; kas zayıflaması; LncRNA'lar; mikrodizi; yanık.

Ulus Travma Acil Cerrahi Derg 2016;22(3):224-232 doi: 10.5505/tjtes.20I5.0483। 